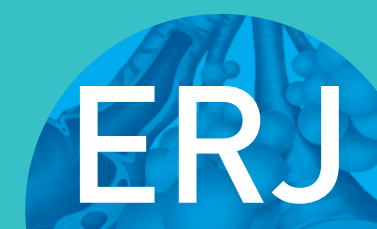

open research
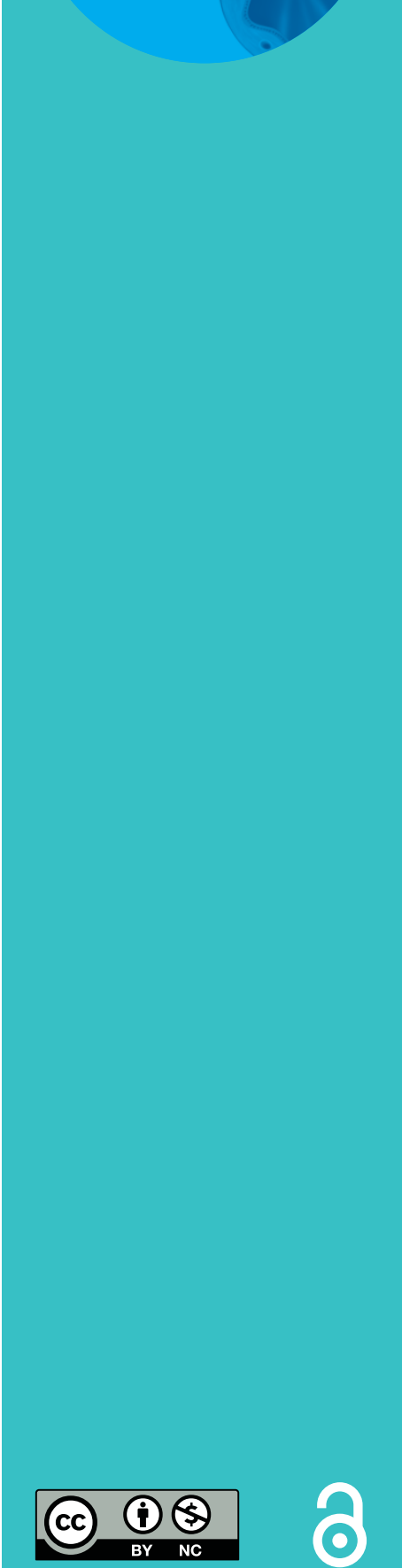

\section{Leukotriene B4 levels in sputum from asthma patients}

\author{
Andrew Higham ${ }^{1}$, Paul Cadden ${ }^{1}$, Thomas Southworth ${ }^{1}$, Matthew Rossall ${ }^{1}$, \\ Umme Kolsum ${ }^{1}$, Simon Lea ${ }^{1}$, Richard Knowles ${ }^{2}$ and Dave Singh ${ }^{1}$
}

Affiliations: ${ }^{1}$ Centre for Respiratory Medicine and Allergy, Institute of Inflammation and Repair, Manchester Academic Health Science Centre, The University of Manchester and University Hospital of South Manchester, NHS Foundation Trust, Manchester, UK. ${ }^{2}$ Respiratory CEDD, GlaxoSmithKline, Stevenage, UK.

Correspondence: Andrew Higham, University of Manchester, Institute of Inflammation and Repair, 2nd Floor, ERC, Southmoor Road, Manchester, M23 9LT, UK. E-mail: Andrew.Highamamanchester.ac.uk

ABSTRACT Poor asthma control is associated with increased airway neutrophils. Leukotriene B4 (LTB4) is a potent neutrophil chemoattractant. We examined the levels of LTB4 levels in the sputum of asthma patients and the relationship with disease severity.

47 asthma patients (categorised according to Global Initiative for Asthma treatment stage) and 12 healthy controls provided sputum samples that were processed first with PBS to obtain supernatants and secondly with dithiothreitol (DTT) to obtain supernatants. LTB4 levels were determined by ELISA.

LTB4 levels were significantly higher in step 1 (steroid naive) and step 3 (inhaled corticosteroid (ICS) plus long acting $\beta$-agonist) patients than step 2 patients (ICS alone) ( $\mathrm{p}=0.02$ and $\mathrm{p}=0.01$, respectively). There was very good correlation when comparing PBS processed to DTT processed supernatants.

High LTB4 levels were found in the sputum of asthmatics at step 3 despite ICS use.

@ERSpublications

The levels of LTB4 are increased in the sputum of subgroups of asthma patients http://ow.ly/Xu6I303jVb5

This article has supplementary material available from openres.ersjournals.com

Received: Nov 162015 | Accepted after revision: July 072016

Support statement: This report is independent research supported by National Institute for Health Research South Manchester Respiratory and Allergy Clinical Research Facility at University Hospital of South Manchester NHS Foundation Trust. The views expressed in this publication are those of the authors and not necessarily those of the NHS, the National Institute for Health Research or the Department of Health. Funding information for this article has been deposited with the Open Funder Registry.

Conflict of interest: Disclosures can be found alongside this article at openres.ersjournals.com

Copyright $\odot$ ERS 2016. This article is open access and distributed under the terms of the Creative Commons Attribution Non-Commercial Licence 4.0. 


\section{Introduction}

Asthma is characterised by variable airflow obstruction, bronchial hyperreactivity and airway inflammation [1]. Asthma is a heterogeneous disease comprised of subgroups of patients with distinct phenotypic characteristics $[2,3]$. Induced sputum sampling has been used to define such phenotypes, with patients being classified as having neutrophilic, eosinophilic, mixed or paucigranulocytic airway disease [4].

Neutrophilic asthma is associated with more severe disease [5, 6]. In addition, neutrophil numbers increase in the airways during exacerbations [7]. Neutrophils secrete reactive oxygen species and proteases which may cause tissue damage resulting in airway remodelling. Drugs that target neutrophil activity may provide clinical benefits to patients with neutrophilic asthma.

Leukotriene B4 (LTB4) is produced from the metabolism of arachadonic acid by 5-lipoxygenase [8]. LTB4 is produced by a variety of cells, including neutrophils and macrophages, and acts via LTB4 receptor 1 (BLT1) and BLT2 G protein receptors [9, 10]. LTB4 is a neutrophil chemoattractant [11] which may play an important role in neutrophilic asthma [10, 11]. LTB4 is also involved in CD8 lymphocyte recruitment and interleukin-13 driven inflammation [12]. The role of cysteinyl-leukotrienes (LTC4, LTD4 and LTE4) in asthma has been well described [13, 14], while the relationship between LTB4 and neutrophilic airway inflammation in asthma is less clear.

In several studies, LTB4 levels have been measured in the supernatant of dithiothreitol (DTT) processed sputum from patients with asthma [15-19]. Only one of these studies reported increased LTB4 levels in asthma; VACHIER et al. [18] demonstrated significantly higher levels of sputum supernatant LTB4 in severe asthmatics compared to healthy controls. DTT is a mucolytic that is used in sputum processing to reduce viscosity so that good quality cytospins can be obtained for cell counts. However, DTT interferes with immunosassays including those for LTB4 [20, 21]. Furthermore, LTB4 levels in DTT processed sputum are unstable, in contrast to non-DTT processed sputum where stable LTB4 levels are observed [22]. A two-step sputum processing procedure involving PBS processing for DTT-free supernatant collection followed by DTT processing for cell counts allows the collection of DTT-free supernatant and good quality cytospins.

We will investigate LTB4 levels in PBS processed sputum supernatants of patients with asthma compared to controls, and the relationships between LTB4 levels and disease severity.

\section{Methods}

Subjects

47 patients with asthma and 12 healthy controls were recruited. Patients with asthma were categorised into groups using the Global Initiative for Asthma (GINA) treatment classification guidelines [23] as follows. Step 1: short-acting $\beta$-agonist; step 2: inhaled corticosteroids (ICS); step 3: ICS plus long-acting $\beta$-agonist.

Inclusion criteria were physician diagnosis of asthma prior to the age of 40 years with current symptoms as defined by guidelines [23], age $\geqslant 18$ years. Patients using ICS were recruited if they had been using this treatment for $>6$ months. Subjects were excluded if they received oral corticosteroids, montelukast or omalizumab. Subjects with a history of an asthma exacerbation within 4 weeks, defined as worsening of asthma symptoms requiring a change in therapy by a physician or a change in regular asthma therapy, were excluded. Subjects who experienced symptoms of a respiratory tract infection within 4 weeks or with any other respiratory conditions were excluded. All subjects were never-smokers. Written informed consent was obtained and the local ethics committee approved the study.

\section{Study design}

Subjects attended for a single visit, where medical history, physical examination and asthma control questionnaire score (ACQ) [24] were performed first, followed by (in strict order) exhaled nitric oxide (eNO), spirometry and reversibility (after $200 \mu \mathrm{g}$ salbutamol), and sputum induction with hypertonic saline.

\section{Spirometry and eNO measurements}

Spirometry was performed using a dry wedge spirometer (Vitalograph, Maids Moreton, UK) according to standard guidelines [25]. eNO was measured at a $50 \mathrm{~mL} \cdot \mathrm{s}^{-1}$ flow rate (Niox; Aerocrine, Solna, Sweden) with subjects exhaling slowly into the metre; results are reported as the mean of three readings in parts per billion.

\section{Induced sputum}

Sputum was induced using 3\%, $4 \%$ and 5\% saline, inhaled in sequence for $5 \mathrm{~min}$, for a maximum of 15 min via an ultrasonic nebuliser (EASYneb II; Flaemnouva, Desenzano del Garda, Italy). To minimise contamination of saliva, all subjects were instructed to thoroughly rinse their mouth with distilled water and perform coughing prior to sputum expectoration. Sputum plugs were isolated from saliva component. For PBS processed sputum, eight volumes of PBS per weight of sputum were added, the sample was 
vortexed and then rocked for $15 \mathrm{~min}$ before being centrifuged at $400 \mathrm{~g}$ for $10 \mathrm{~min}$ at $4^{\circ} \mathrm{C}$. Four volumes of PBS supernatant were removed and stored at $-80^{\circ} \mathrm{C}$. For DTT processed sputum, four volumes of $0.1 \%$ DTT were added, the sample was vortexed and rocked before being filtered and centrifuged. The resulting supernatant was removed and stored at $-80^{\circ} \mathrm{C}$. The remaining cell pellet was re-suspended in PBS prior to cytospin preparation. Cytospins were air dried for $30 \mathrm{~min}$ and stained using RapiDiff (Triangle, Skelmersdale, UK). A differential cell count was conducted on a total of 400 cells by two observers.

\section{Supernatant analysis}

The levels of LTB4 in sputum supernatants of PBS and DTT processed sputum were measured by ELISA according to manufacturer's instructions (Assay Designs, Exeter, UK). This is a competitive immunoassay whereby LTB4 present in the sputum competes with LTB4 which is covalently attached to alkaline phosphatase. Therefore, the intensity of the colorimetric reaction is inversely proportional to the amount of LTB4 present in the sputum. The lower limit of detection was $11.7 \mathrm{pg} \cdot \mathrm{mL}^{-1}$ and the upper limit of detection was $3000 \mathrm{pg} \cdot \mathrm{mL}^{-1}$. Samples were analysed neat and as a series of dilutions. This was conducted to mitigate the risk of samples not falling in the range of the standard curve. Therefore, any samples over $3000 \mathrm{pg} \cdot \mathrm{mL}^{-1}$ will have been diluted to fall within the range of the standard curve.

\section{Statistical analysis}

The sample size for this study was determined a priori to be at least 12 subjects in each category based on previous studies using induced sputum $[17,24,26]$. It was not possible to perform a formal power calculation, as there is no published LTB4 data from PBS processed sputum supernatant. Normality of data was assessed using the Kolmogorov-Smirnov test. Comparisons between groups were made using ANOVA followed by unpaired t-tests for parametric data and the Kruskal-Wallis test followed by the Mann-Whitney U-test for non-parametric data. Confidence intervals of median data were calculated. Univariate analysis was performed to assess the relationship between raw LTB4 levels and measurements of asthma severity and airway inflammation. A partial correlation analysis controlling for sex, age, and ICS use was also conducted to assess the relationship between raw LTB4 levels and measurements of asthma severity and airway inflammation. The LTB4 dataset was not normally distributed; therefore, a Spearman's rank correlation was used. These analyses were conducted using GraphPad software (San Diego, CA, USA), apart from the partial correlation analysis which was conducted using IBM SPSS (Armonk, NY, USA). A p-value $<0.05$ was considered significant.

\section{Results}

\section{Demographics}

Patient demographics and lung function of 12 healthy controls, 17 step 1 patients, 14 step 2 patients and 16 step 3 patients are shown in table 1 . There were differences in forced expiratory volume in $1 \mathrm{~s}$ (FEV 1 ) \% predicted and ACQ between groups. FEV1 \% predicted was lower in step 2 and 3 patients compared to healthy controls and step 1 patients, while ACQ was significantly worse in step 3 patients compared to step 1 patients $(\mathrm{p}=0.03)$. eNO levels were significantly higher in step 1 and 2 patients compared to controls $(\mathrm{p}=0.02$ and $\mathrm{p}=0.04$ respectively), but there were no differences between asthma groups.

\section{Sputum cell counts}

Sputum differential cell counts are shown in table 2. The eosinophil percentage was significantly higher in the step 2 group compared to healthy controls $(\mathrm{p}=0.003)$. Similarly, there was a significant increase in the absolute number of eosinophils in the step 2 and 3 groups compared to healthy controls ( $\mathrm{p}=0.002$ and $\mathrm{p}=0.02$, respectively), and in the step 2 group compared to the step 1 group $(\mathrm{p}=0.04)$. There was a numerical increase in the number of neutrophils in the step 2 and 3 groups compared to step 1 and healthy controls but this did not reach statistical significance.

\section{Supernatant LTB4 levels}

PBS processed sputum

LTB4 levels measured in 59 PBS processed sputum supernatants are shown in figure 1. LTB4 levels were significantly different when comparing the four groups of patients (Kruskal-Wallis $\mathrm{p}=0.03$ ) (figure 1). Pairwise comparisons between groups showed that LTB4 levels in step 1 (median (95\% CI) 1532 (750-3047) pg.mL $\mathrm{mL}^{-1}$ ) and step 3 (median (95\% CI) $1565(833-4274) \mathrm{pg} \cdot \mathrm{mL}^{-1}$ ) patients were numerically higher than healthy controls (median $=879 \mathrm{pg} \cdot \mathrm{mL}^{-1} 95 \%$ CI $520-1852 \mathrm{pg} \cdot \mathrm{mL}^{-1}$ ) but these differences did not reach statistical significance ( $\mathrm{p}=0.2$ and $\mathrm{p}=0.07$, respectively). There were significantly higher levels of LTB4 in the sputum of step 1 and 3 patients compared to step 2 patients $(\mathrm{p}=0.02$ and $\mathrm{p}=0.015$, respectively). 


\begin{tabular}{|c|c|c|c|c|c|}
\hline \multirow[t]{2}{*}{ Clinical characteristics } & \multirow[t]{2}{*}{ Healthy } & \multicolumn{3}{|c|}{ GINA } & \multirow[t]{2}{*}{ p-value } \\
\hline & & Step 1 & Step 2 & Step 3 & \\
\hline Subjects & 12 & 17 & 14 & 16 & \\
\hline Male/female & $5 / 7$ & $12 / 5$ & $7 / 7$ & $10 / 6$ & $>0.05$ \\
\hline Daily ICS dose" mcg & 0.0 & 0.0 & $400 \pm 218^{\Uparrow}$ & $1050 \pm 572$ & $<0.001$ \\
\hline Age years & $36 \pm 10$ & $33 \pm 11$ & $46 \pm 13$ & $41 \pm 16$ & $>0.05$ \\
\hline Age at asthma onset years & NA & $8 \pm 10$ & $15 \pm 16$ & $12 \pm 18$ & $>0.05$ \\
\hline Asthma duration years & NA & $26 \pm 11$ & $31 \pm 163$ & $33 \pm 16$ & $>0.05$ \\
\hline FEV $1 \%$ predicted & $106 \pm 13^{1,+}$ & $97 \pm 16^{\text {ต, }+}$ & $83 \pm 21$ & $81 \pm 17$ & 0.001 \\
\hline FVC $\%$ predicted & $112 \pm 150^{9}$ & $109 \pm 130^{9}$ & $101 \pm 16$ & $100 \pm 15$ & $>0.05$ \\
\hline Reversibility mL & $50 \pm 138 *, 1,+$ & $278 \pm 176$ & $402 \pm 274^{\text {ๆ }}$ & $201 \pm 217$ & $<0.001$ \\
\hline Reversibility \% & $1.5 \pm 4.6^{* .9},+$ & $8.1 \pm 5.9^{+}$ & $17.8 \pm 15.2$ & $8.5 \pm 8.8$ & 0.001 \\
\hline FeNO ppb & $22 \pm 19^{*,+}$ & $40 \pm 23$ & $36 \pm 23$ & $29 \pm 21$ & $>0.05$ \\
\hline ACQ-7 Score & NA & $0.8 \pm 0.4^{\pi}$ & $1.2 \pm 0.7$ & $1.5 \pm 1.0$ & $<0.001$ \\
\hline \multicolumn{6}{|c|}{$\begin{array}{l}\text { Data are presented as mean } \pm \mathrm{SD} \text {, unless otherwise stated. GINA: Global Initiative for Asthma; ICS: inhaled } \\
\text { corticosteroid; FEV1: forced expiratory volume in } 1 \mathrm{~s} \text {; FVC: forced vital capacity; FeNO: exhaled nitric oxide } \\
\text { fraction; ACQ: asthma control questionnaire; NA; not applicable. Data were analysed by one-way ANOVA. } \\
{ }^{\#} \text { : beclomethasone equivalent dose; }{ }^{\natural}: p<0.05 \text { versus GINA step } 3{ }^{+}{ }^{+}: p<0.05 \text { versus GINA step } 2 .^{*}: p<0.05 \\
\text { versus GINA step 1. }\end{array}$} \\
\hline
\end{tabular}

\section{DTT processed sputum}

LTB4 was measured in DTT processed sputum from 52 out of a possible 59 patients (12 healthy controls, 16 step 1 patients, 12 step 2 patients and 12 step 3 patients); there was insufficient sample available from seven patients. LTB4 levels were significantly different when comparing the four groups (Kruskal-Wallis $\mathrm{p}=0.03$ ) (figure 1), with the pattern of results being very similar to the PBS processed data. LTB4 levels were significantly higher in the step 3 group (median (95\% CI) $\left.2582(1279-5337) \mathrm{pg} \cdot \mathrm{mL}^{-1} ; \mathrm{p}=0.03\right)$ and numerically higher in the step 1 group (median (95\% CI) $2732(1321-5151) \mathrm{pg} \cdot \mathrm{mL}^{-1}$; p=0.08) compared to healthy controls (median $\left.(95 \% \mathrm{CI}) 953(595-3624) \mathrm{pg} \cdot \mathrm{mL}^{-1}\right)$. There were significantly higher LTB4 levels in step 1 and 3 patients compared to step 2 patients ( $\mathrm{p}=0.04$ and $\mathrm{p}=0.03$, respectively).

There was very good correlation when comparing results from PBS and DTT processed sputum in all patients $(\mathrm{r}=0.98, \mathrm{p}<0.001)$ and step 1 patients only $(\mathrm{r}=0.99 \mathrm{p}<0.001)$ (figure 2$)$. Data combined from all groups shows LTB4 levels in PBS processed sputum were significantly lower compared to DTT processed sputum $(\mathrm{p}<0.0001)$.

\section{LTB4 levels and asthma severity}

Univariate analysis and partial correlation analysis found no significant associations ( $p>0.05)$ between LTB4 levels in PBS processed sputum and measurements of asthma severity (FEV1\% predicted, ACQ or eNO).

\section{TABLE 2 Induced sputum characteristics of the study population}

\begin{tabular}{|c|c|c|c|c|c|}
\hline \multirow[t]{2}{*}{ Induced sputum } & \multirow[t]{2}{*}{ Healthy } & \multicolumn{3}{|c|}{ GINA } & \multirow[t]{2}{*}{ p-value } \\
\hline & & Step 1 & Step 2 & Step 3 & \\
\hline Subjects & 8 & 14 & 14 & 13 & \\
\hline Neutrophils \% & $37.2 \pm 21.4$ & $44.6 \pm 31.3$ & $52.6 \pm 25.2$ & $55.0 \pm 24.8$ & $>0.05$ \\
\hline Eosinophils \% & $0(0-2.3)^{\#}$ & $0.8(0-6.8)$ & $1.3(0-24.3)$ & $2.0(0-28.8)$ & 0.07 \\
\hline Macrophages \% & $57.5 \pm 24.9$ & $50.7 \pm 32.5$ & $40.6 \pm 24.8$ & $38.1 \pm 23.2$ & $>0.05$ \\
\hline Absolute eosinophil count $\times 10^{6} \cdot \mathrm{g}^{-1}$ sputum & $0(0-0.04)^{\#, 9}$ & $0.03(0-0.3)^{\#}$ & $0.07(0-0.4)$ & $0.04(0-0.7)$ & 0.007 \\
\hline Absolute macrophage count $\times 10^{6} \cdot \mathrm{g}^{-1}$ sputum & $1.5(0.5-3.9)$ & $0.8(0.5-1.4)$ & $0.9(0.5-1.9)$ & $1.0(0.6-1.6)$ & $>0.05$ \\
\hline Absolute lymphocytes count $\times 10^{6} \cdot \mathrm{g}^{-1}$ sputum & $0.01(0-0.2)$ & $0(0-0.2)^{\#, \emptyset}$ & $0.02(0-0.3)$ & $0.02(0-0.2)$ & 0.04 \\
\hline
\end{tabular}

Data are presented median (interquartile range) or mean $\pm S D$, unless otherwise stated. For absolute eosinophil count geometric mean was used. GINA: Global Initiative for Asthma. Parametric data analysed by one-way ANOVA, non-parametric data analysed by Kruskal-Wallis. ${ }^{\#}: p<0.05$ versus GINA step 2; ๆ : $p<0.05$ versus GINA step 3. 

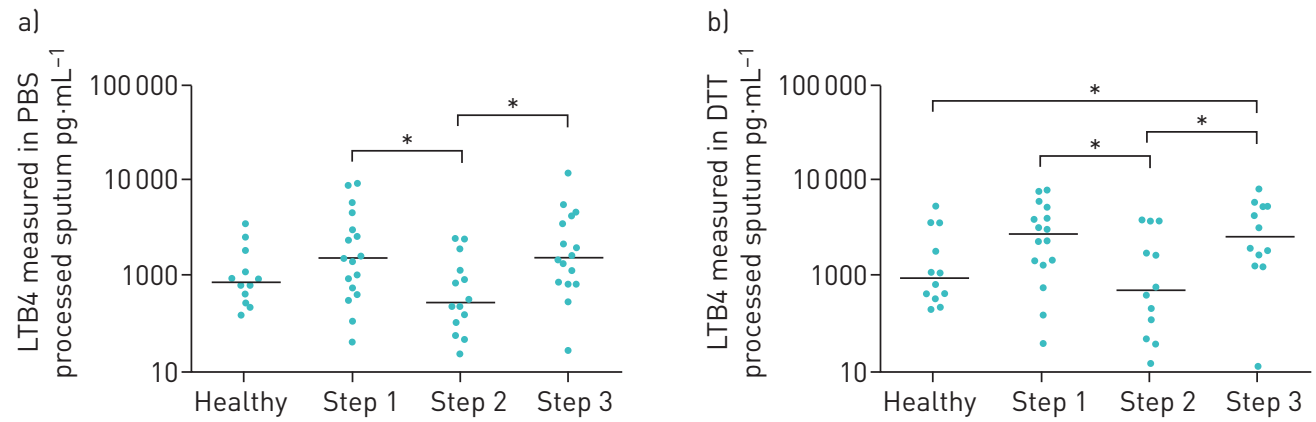

FIGURE 1 A comparison between leukotriene B4 (LTB4) levels in the sputum of healthy controls and asthmatics. LTB4 was measured in a) PBS processed sputum supernatants from 12 healthy controls and 47 asthmatics (step 1: $n=17$, step 2: $n=14$, step 3: $n=16$ ) and b) dithiothreitol (DTT) processed sputum supernatants from 12 healthy controls and 40 asthmatics (step 1: $n=16$, step 2: $n=12$, step 3: $n=12$ ) by ELISA. *: $p<0.05$, significant difference in LTB4 levels.

\section{LTB4 levels and sputum cellularity}

The asthma cohort was sub-grouped based on sputum cellularity as follows. Neutrophilic: neutrophils $>60 \%$, eosinophils $<3 \%$; eosinophilic: eosinophils $>3 \%$, neutrophils $<60 \%$; mixed: neutrophils $>60 \%$, eosinophils $>3 \%$; paucigranulocytic: neutrophils $<60 \%$, eosinophils $<3 \%[4,27]$. There were no significant differences in LTB4 levels between groups in both PBS and DTT processed sputum (figure 3). In addition, there was no correlation between the number of neutrophils or eosinophils and LTB4 levels (supplementary material).

\section{Discussion}

We observed significant differences between asthma subgroups in the levels of sputum LTB4. GINA step 1 and 3 patients had the highest LTB4 levels; this was observed using both PBS and DTT processed sputum.

LTB4 levels in step 1 and 3 patients were significantly higher than step 2 patients. The step 1 and 3 results were also numerically higher than healthy controls, showing trends towards statistical significance. On a group basis, the highest LTB4 levels were in steroid naïve asthma patients, or those on higher ICS doses. However, the individual values overlapped considerably between groups. It appears that there is a subgroup of asthma patients with LTB4 levels greater than those found in healthy controls, and that these individuals are generally either step 1 or 3 patients.

We have observed numerically higher levels of LTB4 in step 1 patients compared to healthy subjects. Similarly, increased LTB4 levels have also been observed in the exhaled breath condensate of mild asthmatics compared to healthy controls $[28,29]$. The role of LTB4 in mild asthma is unknown, but these observations suggest a role for LTB4 levels in asthma pathophysiology even in mild patients.

The use of ICS appears to reduce LTB4 levels in step 2 patients, suggesting that the production of LTB4 is part of a "corticosteroid sensitive" inflammatory milieu in this group of patients. Similarly, it has been reported that ICS intervention in steroid naïve asthmatics reduced sputum LTB4 levels [19]. In contrast, step 3 patients have higher levels of LTB4 despite ICS use. This is an important finding of the study which supports previous observations that asthma patients with more severe disease respond poorly to ICS therapy. The underlying

a)

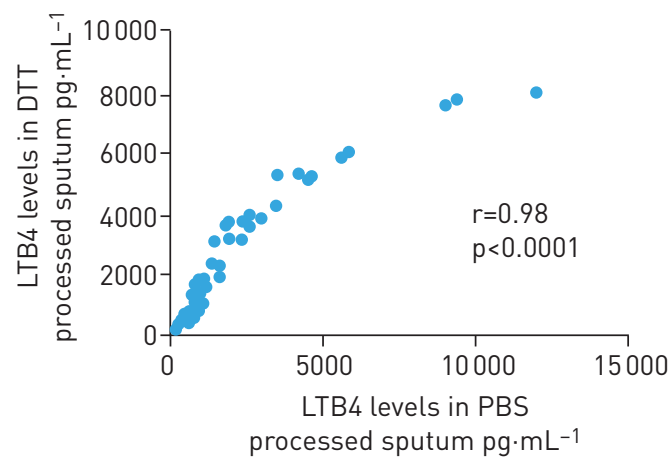

b)

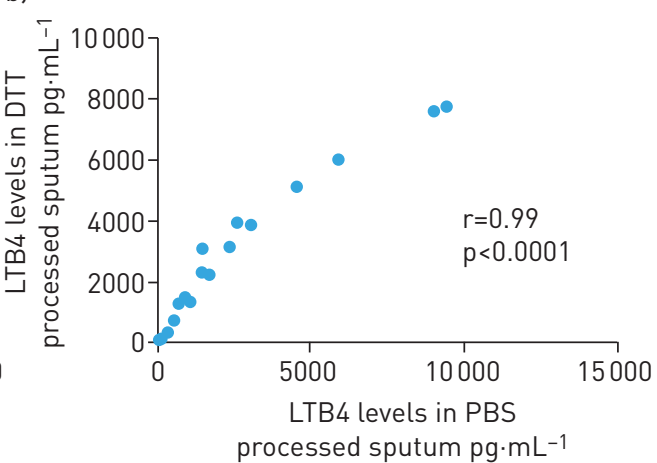

FIGURE 2 Univariate Spearman's analysis between leukotriene B4 (LTB4) levels measured in PBS and dithiothreitol (DTT) processed sputum. Analysis was performed in sputum supernatants isolated from a) all study subjects (12 healthy controls and 40 asthmatics: (step 1: $n=16$, step 2: $n=12$, step $3: n=12$ ) or from b) step 1 only. 
a)

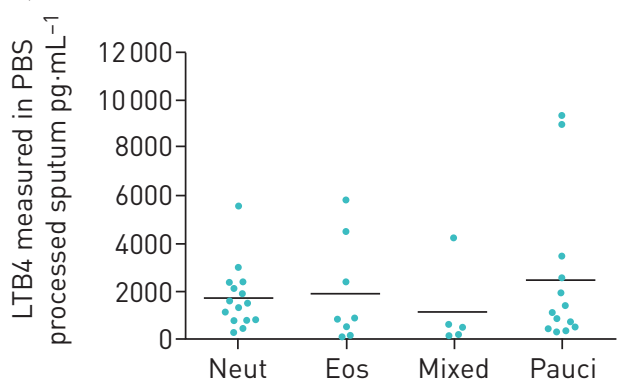

b)

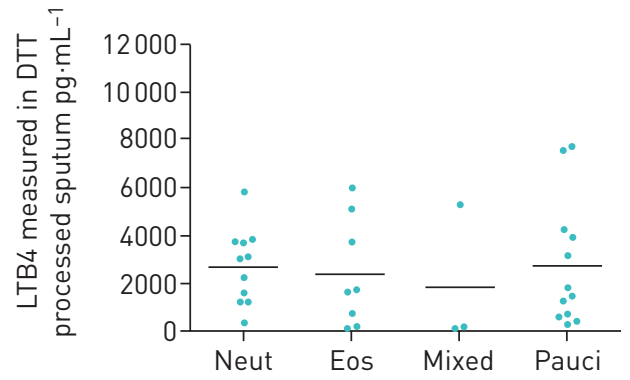

FIGURE 3 Leukotriene B4 (LTB4) levels in asthmatic patients when stratified according to the sputum cellularity. LTB4 levels were measured in a) PBS processed sputum supernatants and b) dithiothreitol (DTT) processed sputum supernatants from patients who were neutrophilic (neut) (PBS: $n=15, D T T: n=11$ ), eosinophilic (eos) (PBS: $n=8$, DTT: $n=8$ ), mixed (PBS: $n=5$, DTT: $n=3$ ) or paucigranulocytic (pauci) (PBS: $n=13, D T T: n=12$ ).

mechanisms are not fully understood, but we have previously observed reduced corticosteroid inhibition of LPS stimulated cytokine production from alveolar macrophages from step 3 and 4 patients compared to step 1 and 2 patients. Similarly, LPS stimulated LTB4 production from the alveolar macrophages of healthy controls and non-severe asthmatics is more sensitive to corticosteroids compared to severe asthmatics [30]. These in vitro findings suggest that macrophage-derived LTB4 may contribute to the presence of LTB4 in the airways of some step 3 patients despite ICS use.

The step 3 patients had similar lung function compared to step 2 patients, but had worse asthma control despite using a higher ICS dose. Failing to reduce LTB4 in these patients may contribute to the ongoing inflammatory process. However, overall we found no relationship between LTB4 levels and asthma control or FEV1. To further understand the role of LTB4 in asthma, investigation using a group of more severe asthmatics is required.

LTB4 is a neutrophil chemoattractant $[31,32]$. However, we observed no correlation between the levels of LTB4 and sputum neutrophils in asthma patients. Similarly, administration of an oral 5-lipoxygenase-activating protein inhibitor did not reduce the percentage of sputum neutrophils or improve clinical endpoints in asthmatics, despite reducing levels of LTB4 [33]. These findings suggest that LTB4 is not the dominant mediator for neutrophilic inflammation in asthma.

We observed a strong association between LTB4 levels in PBS and DTT processed sputum. There was a significant difference between the absolute measurements obtained by the different methods; this was expected in the light of previous findings that DTT can interfere with immunoassays and affect LTB4 stability in the sputum supernatant matrix [21]. The assay used in this study is a competitive immunoassay. This means the intensity of the colorimetric reaction is inversely proportional to the quantity of LTB4 present. We suggest that DTT interferes with the stability of the standard LTB4 used in the assay, thus reducing the interaction between the LTB4 analyte and the LTB4 antibody. Due to the competitive nature of this assay, reduced binding of the LTB4 analyte results in a reduced signal which translates as more LTB4 present in the DTT processed sample. Nevertheless, the same pattern of differences between asthma groups was apparent using both DTT and PBS processed sputum.

A strength of the study was the use of clearly defined subgroups of asthmatic patients based on the severity of disease, treatment regimens and induced sputum cell counts. This allowed us to investigate the relationship between the levels of LTB4 and different disease parameters. However, the lack of a more severe asthma group is a limitation.

Induced sputum is a noninvasive method which allows examination of airway inflammation. It may be used to identify suitable biomarkers to subgroup asthma patients to help develop novel therapies. There are limitations to the method, such as the contamination of sputum with saliva. We have employed a method to reduce this risk but cannot fully rule out the contamination of the sputum.

In conclusion, our results indicate that sputum LTB4 levels are raised in a subgroup of asthma patients. Interestingly, these patients were confined to GINA steps 1 and 3, and not step 2. This suggests a role for LTB4 in asthma pathophysiology, but not in all patients. The concept of both asthma phenotypes and endotypes is increasingly recognised, $[2,34]$. Our results indicate sputum LTB4 may be used to identify a subgroup of patients that may benefit from specific therapies targeted against LTB4 production. 


\section{References}

$1 \quad$ Holgate ST. Pathogenesis of asthma. Clin Exp Allergy 2008; 38: 872-897.

2 Wenzel SE. Asthma: defining of the persistent adult phenotypes. Lancet 2006; 368: 804-813.

3 Moore WC, Meyers DA, Wenzel SE, et al. Identification of asthma phenotypes using cluster analysis in the Severe Asthma Research Program. Am J Respir Crit Care Med 2010; 181: 315-323.

4 Simpson JL, Scott R, Boyle MJ, et al. Inflammatory subtypes in asthma: assessment and identification using induced sputum. Respirology 2006; 11: 54-61.

5 Woodruff PG, Khashayar R, Lazarus SC, et al. Relationship between airway inflammation, hyperresponsiveness, and obstruction in asthma. J Allergy Clin Immunol 2001; 108: 753-758.

6 Moore WC, Hastie AT, Li X, et al. Sputum neutrophil counts are associated with more severe asthma phenotypes using cluster analysis. J Allergy Clin Immunol 2014; 133: 1557-1563.

7 Fahy JV, Kim KW, Liu J, et al. Prominent neutrophilic inflammation in sputum from subjects with asthma exacerbation. J Allergy Clin Immunol 1995; 95: 843-852.

8 Wenzel SE. Arachidonic acid metabolites: mediators of inflammation in asthma. Pharmacotherapy 1997; 17: 3S-12S.

9 Peters-Golden M, Henderson WRJr. Leukotrienes. N Engl J Med 2007; 357: 1841-1854.

10 Izumi T, Yokomizo $\mathrm{T}$, Obinata $\mathrm{H}$, et al. Leukotriene receptors: classification, gene expression, and signal transduction. J Biochem 2002; 132: 1-6.

11 Corhay JL, Henket M, Nguyen D, et al. Leukotriene B4 contributes to exhaled breath condensate and sputum neutrophil chemotaxis in COPD. Chest 2009; 136: 1047-1054.

12 Gelfand EW, Dakhama A. CD8+ T lymphocytes and leukotriene B4: novel interactions in the persistence and progression of asthma. J Allergy Clin Immunol 2006; 117: 577-582.

13 Busse W, Kraft M. Cysteinyl leukotrienes in allergic inflammation: strategic target for therapy. Chest 2005; 127: $1312-1326$.

14 Leigh R, Vethanayagam D, Yoshida M, et al. Effects of montelukast and budesonide on airway responses and airway inflammation in asthma. Am J Respir Crit Care Med 2002; 166: 1212-1217.

15 Gaber F, Daham K, Higashi A, et al. Increased levels of cysteinyl-leukotrienes in saliva, induced sputum, urine and blood from patients with aspirin-intolerant asthma. Thorax 2008; 63: 1076-1082.

16 Pohl D, Beier J, Buhl R, et al. Effect of histamine and adenosine 5'-monophosphate provocation on sputum neutrophils and related mediators in atopic patients. Ann Allergy Asthma Immunol 2005; 95: 197-203.

17 Kostikas K, Gaga M, Papatheodorou G, et al. Leukotriene B4 in exhaled breath condensate and sputum supernatant in patients with COPD and asthma. Chest 2005; 127: 1553-1559.

18 Vachier I, Bonnans C, Chavis C, et al. Severe asthma is associated with a loss of LX4, an endogenous anti-inflammatory compound. J Allergy Clin Immunol 2005; 115: 55-60.

19 Basyigit I, Yildiz F, Ozkara SK, et al. Inhaled corticosteroid effects both eosinophilic and non-eosinophilic inflammation in asthmatic patients. Mediators Inflamm 2004; 13: 285-291.

20 Wang F, He B. The effect of dithiothreitol on chemotactic factors in induced sputum of chronic obstructive pulmonary disease patients. Respiration 2009; 78: 217-222.

21 Woolhouse IS, Bayley DL, Stockley RA. Effect of sputum processing with dithiothreitol on the detection of inflammatory mediators in chronic bronchitis and bronchiectasis. Thorax 2002; 57: 667-671.

22 Jian W, Edom RW, Xue X, et al. Quantitation of leukotriene B(4) in human sputum as a biomarker using UPLC-MS/MS. J Chromatogr B Analyt Technol Biomed Life Sci 2013; 932: 59-65.

23 Bateman ED, Hurd SS, Barnes PJ, et al. Global strategy for asthma management and prevention: GINA executive summary. Eur Respir J 2008; 31: 143-178.

24 Juniper EF, Bousquet J, Abetz L, et al. Identifying "well-controlled" and "not well-controlled" asthma using the Asthma Control Questionnaire. Respir Med 2006; 100: 616-621.

25 Miller MR, Crapo R, Hankinson J, et al. General considerations for lung function testing. Eur Respir J 2005; 26: $153-161$.

26 Tak T, Hilvering B, Tesselaar K, et al. Similar activation state of neutrophils in sputum of asthma patients irrespective of sputum eosinophilia. Clin Exp Immunol 2015; 182: 204-212.

27 Simpson JL, McElduff P, Gibson PG. Assessment and reproducibility of non-eosinophilic asthma using induced sputum. Respiration 2010; 79: 147-151.

28 Montuschi P, Barnes PJ. Exhaled leukotrienes and prostaglandins in asthma. J Allergy Clin Immunol 2002; 109: 615-620.

29 Kazani S, Planaguma A, Ono E, et al. Exhaled breath condensate eicosanoid levels associate with asthma and its severity. J Allergy Clin Immunol 2013; 132: 547-553.

30 Bhavsar PK, Levy BD, Hew MJ, et al. Corticosteroid suppression of lipoxin A4 and leukotriene B4 from alveolar macrophages in severe asthma. Respir Res 2010; 11: 71.

31 McDonald B, Kubes P. Chemokines: sirens of neutrophil recruitment - but is it just one song? Immunity 2010; 33: $148-149$.

32 Afonso PV, Janka-Junttila M, Lee YJ, et al. LTB4 is a signal-relay molecule during neutrophil chemotaxis. Dev Cell 2012; 22: 1079-1091.

33 Chaudhuri R, Norris V, Kelly K, et al. Effects of a FLAP inhibitor, GSK2190915, in asthmatics with high sputum neutrophils. Pulm Pharmacol Ther 2014; 27: 62-69.

34 Lotvall J, Akdis CA, Bacharier LB, et al. Asthma endotypes: a new approach to classification of disease entities within the asthma syndrome. J Allergy Clin Immunol 2011; 127: 355-360. 